\title{
CONHECIMENTO EM HIV/AIDS DE 1998 A 2005: ESTUDOS PUBLICADOS EM PERIÓDICOS DE ENFERMAGEM
}

Knowledge in HIV/ AIDS from 1998 to 2005:

Studies published in Nursing Periodicals

Conocimiento en HIV/ SIDA de 1998 a 2005:

Estudios publicados en Periódicos de Enfermería

Maria Alix Leite Araújo Thelma Leite de Araújo Marta Maria Coelho Damasceno

\begin{abstract}
Resumo
A pesquisa em enfermagem contribui para o fortalecimento da profissão e para a elaboração de um corpo de conhecimentos especializados, específicos e atualizados, buscando torná-la de relevância social. Deve ser analisada visando refletir sobre o conhecimento de maneira a torná-lo mais adequado. Teve-se por objetivo analisar pesquisas publicadas em seis periódicos de enfermagem Qualis internacional no período de 1998 a 2005. Foram encontrados 40 estudos, realizados predominantemente na Região Sudeste, derivados de teses e dissertações e de autoria de docentes. A temática privilegiada foi a vivência e convivência dos portadores de HIV/AIDS. Conclui-se pela necessidade de expansão da abrangência das pesquisas, buscando atender as exigências da área.
\end{abstract}

Palavras-chave: Conhecimento. HIV/AIDS. Enfermagem.

\begin{abstract}
The research in nursing contributes for the profession strengthening and for the elaboration of specialized, specific and updated knowledge body, turning it to social relevance. It must be analyzed to ponder about knowledge in a way to make it more suitable. The goal was to analyze researches published in six nursing periodicals - Qualis International from 1998 to 2005. 40 studies were found, mainly accomplished in the Southeastern Region, originated from tests and essays by teachers' authorship. The privileged set of themes was life experience and company of HIV/AIDS carriers. It was decided for the necessity of the researches scope expansion, in order to assist the area demands.
\end{abstract}

\section{Resumen}

La investigación en enfermería contribuye para el for talecimiento de la profesión y para la elaboración de un cuerpo de conocimientos especializados, específicos y actualizados, buscando tornala de importancia social. Debe ser analizada con vistas a reflexionar el conocimiento para tornarlo más adecuado. Se tuvo por objetivo analisar investigaciones publicadas en seis periódicos de enfermería internacional, en el período de 1998 a 2005. Fueron encontrados 40 estudios, realizados predominantemente en la región sureste, derivados de tesis y disertaciones de autoría de docentes. La temática privilegiada fué experiencia y convivencia de los portadores de VIH/SIDA. Se concluye por la necesidad de expansión de la abrangencia de las investigaciones, buscanco atender a los requisitos del área.
Keywords:

Knowledge. HIV/AIDS. Nursing.
Palabras clave:

Conocimiento. HIV/SIDA. Enfermería. 


\section{INTRODUÇÃO}

No mundo atual cada vez mais globalizado e em constantes mudanças, a enfermagem vem tentando acompanhar os avanços científicos e tecnológicos, procurando, continuamente, elaborar novos conhecimentos e repensando a sua prática. Dessa forma, estabelece um movimento dialético entre o seu pensar e 0 fazer, para se fortalecer como ciência. Para isso, precisa produzir um corpo de conhecimentos próprios, coerentes com esse novo contexto social mundial.

"O fazer profissional demanda conhecimentos diversos que, sob distintas perspectivas, coexistem na busca de integrar o homem em suas várias dimensões como, por exemplo: biológica, política, social, filosófica e religiosa ${ }^{1 "}$. Esses conhecimentos se constroem pela necessidade constante de elaboração de respostas a questionamentos que, quando organizados e esquematizados, constituem as pesquisas. $\mathrm{Na}$ enfermagem, o desenvolvimento de pesquisas é uma atividade relativamente recente, por ser uma profissão ainda jovem do ponto de vista científico.

A produção científica em enfermagem iniciou-se, seguindo 0 modelo padrão dominante que era 0 modelo médico mais dirigido a estudos de natureza quantitativa, apoiado em uma abordagem científica empírica que focalizava uma linha de raciocínio linear, observável, objetivo, lógico, analítico e verbal2.

Outras formas de saber eram consideradas conhecimentos meramente especulativos. Somente a partir da segunda metade da década de 80 é que se observa o aparecimento de outras propostas metodológicas, dirigindo as pesquisas em enfermagem para as ciências humanas e sociais ${ }^{1}$.

Um dos motivos que talvez tenha gerado a necessidade de redimensionar as pesquisas em enfermagem pode ter sido o seu próprio objeto de trabalho que é definido como o cuidado. Esse objeto, que abrange aspectos subjetivos, não deve ser trabalhado em pesquisas de caráter positivista.

Nesse sentido, a enfermagem que a cada dia vem expandindo a sua área de atuação e ocupando espaços importantes no mercado de trabalho, demonstra autonomia e maturidade ao adequar o seu objeto de trabalho a linhas metodológicas que melhor 0 expressem, sejam essas quantitativas ou qualitativas.

Pesquisa liga teoria, educação e prática e alimenta constantemente a prática profissional. Para tanto, as formulações teóricas apoiadas em achados de pesquisa podem potencialmente se tornar 0 fundamento da prática de enfermagem ${ }^{3}$. A pesquisa em enfermagem, além de fortalecer a profissão, contribui para a criação de um corpo de conhecimentos especializados, específicos e atualizados, visando torná-la de relevância social.

Muitos estudos foram realizados e publicados por enfermeiros em diversas áreas e, historicamente, essas publicações estiveram mais ligadas às universidades. Atualmente já se observa uma expansão da pesquisa, permitindo um reflexo de sua aplicabilidade na prática, no ensino de graduação e na assistência à saúde de forma mais ampliada 4 .

Dentro da perspectiva de adequar o conhecimento e o desenvolvimento do saber ao que se apresenta na atualidade, vão surgindo novos desafios que precisam ser encarados e inseridos no contexto científico próprio da profissão. Entre esses encontram-se novas doenças, novas tecnologias e novos modos de enfrentamento.

Um dos desafios que os profissionais de saúde, os governos e a comunidade científica enfrentam na atualidade é a Síndrome da Imunodeficiência Humana, a AIDS, considerada um dos mais sérios problemas de saúde pública mundial.

0 preconceito manifestado pelos profissionais de saúde em relação aos portadores dessa síndrome representa um entrave para o cuidado aos doentes e, especialmente, para a prevenção, pois exige um diálogo aberto sobre temas muitas vezes de difícil abordagem.

Os aspectos relacionados as suas formas de transmissão, à velocidade com que a epidemia tem se disseminado na população e às diferenças que vêm ocorrendo no seu perfil epidemiológico ao longo dos anos, sempre exigiram formas de enfrentamento rápidas por parte do governo e da sociedade civil.

A AIDS trouxe consigo a exigência de novos paradigmas de cuidado que os profissionais de saúde, de um modo geral, e os enfermeiros, em particular, ainda procuram desenvolver, apesar de já terem se passado mais de vinte anos de início da epidemia e dos muitos avanços científicos alcançados.

Os enfermeiros, como citado anteriormente, ampliaram, consideravelmente, a abrangência de suas ações não só restringindo-as ao âmbito da assistência hospitalar, mas também a outras áreas de atuação e, em especial, à prevenção. Assim, eles podem contribuir de maneira considerável com o desenvolvimento de novas descobertas científicas e tecnológicas.

A temática HIV/ AIDS é de interesse de pesquisadores da área de enfermagem há vários anos e, junto a um dos órgãos de fomento, esteve incluída na linha de pesquisa que mais solicitou financiamento ${ }^{5}$. A necessidade de refletir e analisar as publicações sobre 
esse tema é um exercício importante para desenvolver a capacidade de avaliação crítica de outros pesquisadores, bem como para trazer, cada vez mais, aperfeiçoamento aos conhecimentos científicos nessa área.

Dois artigos publicados em periódicos de enfermagem brasileiros, no ano de 2002, analisaram os trabalhos em DST e HIV/AIDS destacando as principais temáticas abordadas, os enfoques metodológicos, a área de atuação dos autores, os tipos de estudos e as tendências das pesquisas realizadas por enfermeiros ${ }^{6.7}$.

Diante da relevância do tema e da necessidade de acompanhamento e redimensionamento constante da produção científica na área em questão, objetivou-se, com o presente estudo, analisar as pesquisas realizadas sobre HIV/ AIDS em periódicos de enfermagem brasileiros. Pretendeu-se, ainda, apresentar as tendências das pesquisas desenvolvidas nessa área e, com isso, contribuir para que o corpo de conhecimentos em Enfermagem seja direcionado para responder aos apelos apresentados pela epidemia.

\section{ABORDAGEM METODOLÓGICA}

Trata-se de um estudo bibliográfico ${ }^{8}$, de abordagem quantitativa, que se propôs a avaliar as pesquisas publicadas com a temática HIV/AIDS, em seis periódicos brasileiros classificados como Qualis Internacional B e Qualis Internacional C. Os periódicos selecionados foram: Revista Brasileira de Enfermagem, Acta Paulista de Enfermagem, Revista Gaúcha de Enfermagem, Texto \& Contexto Enfermagem, Revista da Escola de Enfermagem da USP e Revista Latino-Americana de Enfermagem. Propôs analisar os estudos publicados nos anos de 1998 a 2005, que tiveram enfermeiros como um dos autores, independentemente da presença de outras categorias profissionais.

A escolha de tais periódicos ocorreu por estarem indexados em bases de dados de veiculação internacional, provavelmente fonte de pesquisa da comunidade científica. Com certeza trazem contribuições importantes para a formação do corpo de conhecimentos específicos da enfermagem. 0 recorte do período de tempo foi determinado por se tratar de estudos mais recentes e atualizados sobre a temática.

Inicialmente, foi realizado o levantamento dos artigos na BIREME, tendo como fonte os periódicos selecionados. Em seguida, utilizou-se o banco de dados da Enfermagem (BDENF), com os descritores HIV/ AIDS e ano de publicação. Depois, realizou-se uma leitura preliminar dos títulos e resumos para identificar se estavam relacionados ao objeto do estudo. Foram incluídos somente aqueles que representavam trabalhos de pesquisa, não sendo consideradas outras publicações do tipo relato de experiência, estudo de caso clínico, etc.

Após essa etapa, foram acessados os artigos para leitura completa e levantamento dos dados. A coleta e a análise dos dados foram realizadas nos meses de maio e junho de 2005. Para a coleta dos dados, foi elaborado um formulário e extraído dos artigos os seguintes aspectos: nome do periódico, área de atuação dos autores, vinculação dos estudos (se, a teses de doutorado, a dissertações de mestrado, a trabalhos de disciplina, etc.), local de realização (referente ao local de coleta de dados), temáticas abordadas, tipo e sujeitos dos estudos, técnica de coleta de dados e submissão ao Comitê de Ética.

Para a análise, utilizou-se o Epi-Info 6.0, e os resultados foram apresentados parte em forma descritiva e parte em tabelas.

\section{RESULTADOS}

A partir do levantamento realizado, foram identificados no intervalo compreendido entre os anos de 1998 a 2005, 40 trabalhos publicados nos periódicos selecionados. Houve uma predominância de publicações nos anos 1999 e 2000, com oito e nove artigos publicados respectivamente, e decréscimo no número de publicações a partir de 2001 (Quadro 1). A Revista da Escola de Enfermagem da USP publicou 16 artigos, isto é, $40 \%$ do total. A segunda revista em número de artigos sobre HIV/ AIDS foi a Latino-Americana de Enfermagem com 10 (25\%).

Em geral, os estudos tinham no mínimo dois e no máximo sete autores. Houve predominância de enfermeiros nas autorias, o que se justifica por se tratar de periódicos de enfermagem. Somente três artigos tiveram a participação de outras categorias profissionais (médico, psicólogo e nutricionista). Observou-se que $25(62,5 \%)$ dos artigos são de autoria de docentes de enfermagem, nove $(22,5 \%)$, de docentes e enfermeiros assistenciais e cinco $(12,5 \%)$, de docentes e alunos de pós-graduação ou graduação.

Constatou-se ainda que quatro $(10 \%)$ dos artigos foram extraídos de teses de doutorado, oito $(20 \%)$ de dissertações de mestrado, dois $(5,0 \%)$ de trabalhos de disciplina de cursos de pós-graduação e 26 (65\%), não indicaram vinculação a outro estudo. 
Q uadro 1:

Distribuição das pesquisas realizadas em seis periódicos internacionais de enfermagem por ano de publicação (1998-2005).

\begin{tabular}{|c|c|c|c|c|c|c|c|c|c|c|}
\hline \multirow{2}{*}{ Revista } & \multicolumn{8}{|c|}{ Ano de Publicação } \\
\cline { 2 - 9 } & 1998 & 1999 & 2000 & 2001 & 2002 & 2003 & 20004 & 2005 & TOTAL & $\%$ \\
\hline Esc. Enf. USP & 03 & 03 & 03 & 02 & 01 & 02 & 01 & 01 & 16 & 40 \\
$\begin{array}{c}\text { Lat-am. } \\
\text { Enferm. }\end{array}$ & 02 & 03 & 01 & - & 01 & 01 & - & 02 & 10 & 25 \\
$\begin{array}{c}\text { Bras. } \\
\text { Enferm. }\end{array}$ & 01 & - & 01 & 02 & - & - & - & - & 04 & 10 \\
$\begin{array}{c}\text { Texto } \\
\text { Contexto } \\
\text { Enferm. }\end{array}$ & - & - & 02 & - & - & - & 02 & - & 04 & 10 \\
$\begin{array}{c}\text { Acta Paul. } \\
\text { Enferm. } \\
\text { Gaúcha } \\
\text { Enferm. }\end{array}$ & - & 01 & 01 & - & 01 & - & - & - & 03 & 7,5 \\
TOTAL & 06 & 08 & 09 & 04 & 03 & 03 & 04 & 03 & 40 & 100 \\
\hline
\end{tabular}

\section{Tabela 1:}

Análise dos Estudos publicados em seis periódicos internacionais de enfermagem por Regiões e Estados de realização das pesquisas. (1998-2005)

\begin{tabular}{lccc}
\hline Região & Estado & $\begin{array}{c}\text { No de } \\
\text { trabalhos }\end{array}$ & $\%$ \\
\hline Sudeste & SP & 21 & 77,8 \\
& MG & 05 & 18,5 \\
TOTAL & 01 & 3,7 \\
\hline Nordeste & RN & 27 & 100 \\
& PB & 02 & 42,8 \\
\hline TOTAL & PE & 01 & 14,3 \\
\hline Sul & RS & 02 & 14,3 \\
\hline TOTAL & SC & 02 & 100 \\
\hline TOTAL & & 04 & 100 \\
\hline GERAL & & 40 & 100 \\
\hline
\end{tabular}

Quanto ao local da coleta dos dados, $27(67,5 \%)$ aconteceram na região Sudeste, nove $(22,5 \%)$, na região Nordeste e quatro (10\%), na região Sul. Não foram encontrados no período investigado, artigos cuja coleta de dados tenha ocorrido nas regiões Norte e CentroOeste. Verificou-se que, dos 27 trabalhos da região Sudeste, $21(77,8 \%)$ eram do Estado de São Paulo (Tabela 2).

As principais temáticas abordadas foram: vivência ou convivência com o HIV/ AIDS (14/35\%); conhecimento da população sobre HIVI AIDS (5/12,5\%); prática dos profissionais de saúde (4/10\%); perfil do portador do HIV (4/(10\%); sexualidade do portador do HIV $(3 / 7,5 \%)$; formas de prevenção do HIV/ AIDS (3/7,5\%); percepção do risco para HIV (3/7,5\%); interação DST/HIV (3/7,5\%); diagnóstico de enfermagem $(1 / 2,5 \%)$; prevenção da transmissão vertical do HIV $(1 / 2,5 \%)$. Vale ressaltar que dois artigos não trataram de temática específica, pois realizaram análise da produção cientifica sobre HIV/ AIDS.

A abordagem metodológica predominante nos artigos avaliados foi a qualitativa, encontrada em 22 $(55 \%)$ dos trabalhos publicados. Na caracterização dos sujeitos das pesquisas, encontrou-se que 17 (42,5\%) estudaram portadores de HIV/ AIDS, oito (20\%) profissionais de saúde, dois $(5,0 \%)$, usuários dos serviços de saúde, dois $(5,0 \%)$, adolescentes, dois $(5,0 \%)$, enfermeiros e portadores de HIV/ AIDS, dois $(5,0 \%)$, familiares e portadores do HIV e dois $(5,0 \%)$ parceiros sorodiscordantes, um $(2,5 \%)$, universitários 
da área de saúde, um $(2,5 \% \%)$, universitários e especialistas em HIV/ AIDS e um $(2,5 \%)$ manicures.

Por fim, constatou-se que, em 34 (85\%) estudos a coleta de dados se deu por entrevista, em quatro (10\%), em prontuários e fichas de notificação e dois $(5,0 \%)$ analisaram outros estudos sobre a temática. Somente $23(57,5 \%)$, fizeram referência ao envio da pesquisa ao Comitê de Ética. De um modo geral, os trabalhos que mencionavam tal procedimento ocorreram a partir do ano de 2002, lembrando que, dessa data em diante, as revistas passaram a exigir a aprovação do Comitê de Ética como requisito para a publicação dos artigos.

\section{DISCUSSÃO}

0 interesse demonstrado em publicar sobre a temática em questão se manteve durante 0 período estudado. No entanto, nota-se diminuição no total de publicações a partir de 2001. Tal situação causa estranhamento uma vez que a epidemia de HIV/ AIDS ainda se apresenta como um grave problema de saúde pública no Brasil e no mundo, e portanto um grande desafio para a comunidade. Apesar dos avanços científicos, o número de notificações ainda vem aumentando consideravelmente ao longo dos anos, apresentando inclusive modificações importantes no perfil epidemiológico ${ }^{9}$. Contudo, é preciso considerar a possibilidade de os enfermeiros estarem publicando em outros periódicos que não sejam da área específica da enfermagem.

Destaca-se que a Revista da Escola de Enfermagem da USP manteve regularidade nas publicações sobre o tema, como também apresentou o maior número de artigos publicados. Em artigo citado ${ }^{7}$ que analisa as publicações sobre HIV/ AIDS realizadas no período de 1993 a 1999, a Revista Brasileira de Enfermagem congregou 0 maior número de artigos. Nesse estudo, essa revista aparece em quarto lugar com $10 \%$ das publicações.

A predominância de docentes nas publicações sobre o tema em questão encontra apoio em outros estudos que apontam que há supremacia da docência no que diz respeito às autorias ${ }^{10-11}$. Assim, constatase que essa tendência vem se mantendo ao longo dos anos, não só nessa como em outras áreas estudadas ${ }^{10}$, fortalecendo a idéia equivocada de que a pesquisa é uma atividade restrita à academia. Fazse necessário estimular a participação de profissionais da assistência em projetos de pesquisa, pois talvez, dessa forma, seja possível despertá-los para a importância de uma prática reflexiva e cientificamente embasada ${ }^{3}$. Ainda é muito tímida a participação de outras categorias profissionais nas publicações de enfermagem. Para Reis e Gir ${ }^{7}, 0$ trabalho multiprofissional deve ser estimulado, pois contribui para ampliar 0 entendimento sobre as DST e o HIV, temas que necessitam de uma abordagem interdisciplinar.

Um percentual significativo (35\%) de publicações extraídas de teses, dissertações e trabalhos de disciplina de cursos de pós-graduação demonstra que há uma tendência em divulgar a produção oriunda desses cursos.

Assim como o presente estudo, outros também constataram que na maioria os dados foram coletados na Região Sudeste ${ }^{12}$, mais especificamente no Estado de São Paulo $0^{6-7}$.

Levando-se em consideração os dados epidemiológicos de AIDS no Brasil, esse fato se justifica, pois nessa região ainda se concentra 0 maior número de casos, apesar de a epidemia estar disseminada em todos os estados brasileiros. Dos 362.364 casos notificados ao Ministério da Saúde até junho de 2004, 235.410 estão na Região Sudeste e destes, 153.193 estão no Estado de São Paulo que apresentou o maior número de casos de aids notificados até dezembro de $2003^{9}$.

No entanto, é inquestionável a importância de estudos sobre a epidemia de HIV/ AIDS em locais de baixa prevalência, pois, além de proporcionar uma maior familiaridade com o problema, possibilita 0 desenvolvimento precoce de estratégias de prevenção e assistência. Porém, em locais com alta prevalência eles são imprescindíveis, visto que a epidemia já está instalada e torna ainda mais difícil o controle.

A tendência de se enfocar, entre as temáticas estudadas, a vivência e a convivência das pessoas com o HIV/ AIDS, reitera os resultados do estudo de Sousa et al ${ }^{6}$. Talvez a predominância dessa temática seja decorrente do fato de as pessoas continuarem sendo vítimas de preconceito e discriminação. A compreensão dos sentimentos possibilita 0 atendimento dos aspectos emocionais, preocupação manifestada pela enfermagem. Estudos com abordagem qualitativa são os mais adequados para se estudar sentimentos, e esses foram maioria entre os artigos analisados ${ }^{13}$.

Apesar de haver outros artigos que analisaram a produção científica de enfermagem sobre HIV/ AIDS, este trabalho se justifica porque proporcionou uma análise mais atualizada e concentrada nos últimos oito anos, acerca das publicações sobre 0 tema. Estudos dessa natureza possibilitam o repensar constante dos saberes e o redirecionamento das pesquisas adequando-as ao cenário atual. 


\section{CONSIDERAÇÕES FINAIS}

Ficou evidenciado que a maioria das produções analisadas nos periódicos de enfermagem selecionados ocorreu na Região Sudeste, foi extraída de dissertações ou teses, foi realizada por docentes de cursos de pósgraduação, além de estar voltada para a análise da vivência e convivência de portadores de HIV/ AIDS.

Conclui-se, portanto, que os estudos ainda seguem as mesmas tendências dos anteriores, realizados sobre

\section{Referências}

1. Carvalho EC, Rossi LA. Modelos, estruturas e teorias de enfermagem: a plicação através do processo de enfermagem. In: Garcia TR, Pagliuca LMF, organizadores. A construção do conhecimento em enfermagem: coletânea de trabalhos. Fortaleza (CE): RENE; 1998.

2. Collet N, Schneider JF, Corrêa AK. A pesquisa em enfermagem: avanços e desafios. Rev Bras Enferm 2000 jan/mar; 53(1): 75-80.

3. Wood GL. 0 papel da pesquisa em enfermagem. In: Wood GL, Haber J. Pesquisa em enfermagem: métodos, avaliação, crítica e utilização. Rio de Janeiro (RJ): Guanabara Koogan; 1994.

4. Prado ML, Golbcke FL. Produção do conhecimento em enfermagem no Brasil: as temáticas de investigação. Rev Bras Enferm 2001 jan/ mar; 24(1): 34-42.

5. Leite JL, Trezza MCSF, Santos IACM, Felli VEA. Os projetos de pesquisa de enfermagem no CNPq: seu percurso, suas temáticas, suas aderências (1998-2000). Rev Bras Enferm 2001 jan/mar; 54(1): 81- 97.

7. Reis RK, Gir E. Caracterização da produção científica sobre doenças sexualmente transmissíveis e HIV/AIDS publicados em periódicos de enfermagem no Brasil. Rev Esc Enferm USP 2002 dez; 36 (4): 376-85.

8. Lakatos EM, Marconi MA. Fundamentos de metodologia científica. 4ạ ed revisada e ampliada. São Paulo (SP): Atlas; 1992.

9. Ministério da Saúde (BR). 1a a 26a semanas epidemiológicas: janeiro a junho de 2004. Boletim Epidemiológico de Aids 2004; 1 (1)

10. Guedes MVC, Araújo TL, Damasceno MCD. Análisis de las pesquisas sobre hipertensión ar terial publicadas em periódicos de enfermería. Rev Panamericana Enfermería. 2004 jul/ dic; 2(2): 117-23.

11. Ito EE, Takahashi RT. Publicações sobre ensino em enfermagem na Revista da Escola de Enfermagem da USP. Rev Esc Enferm USP 2005; 4(39): 409-16.

12. Prochnow AG, Leite JL, Olivo VF. Cultura - cultura organizacional: uma análise com enfoque na produção científica da enfermagem. Esc Anna Nery Rev Enferm 2005 ago; 9 (2): 271-77.

13. Minayo MCS. Ciência, técnica e arte: 0 desafio da pesquisa social. In: Deslandes SF, Cruz Neto 0, Minayo MCS,organizadores. Pesquisa social: teoria, método e criatividade. Petrópolis (RJ): Vozes; 1995. a temática. São necessários, e também escassos, trabalhos que abordem 0 desenvolvimento de estratégias de promoção da saúde nos programas de prevenção e assistência aos portadores de HIV/ AIDS. Da mesma forma, poder-se-ia incluir nas pesquisas outros grupos que no momento constituem a população vulnerável, isto é, heterossexuais, e em especial mulheres cujo desdobramento é a contaminação de recém-nascidos durante a gestação, o parto ou 0 aleitamento materno.

\section{Sobre as Autoras}

\section{Maria Alix Leite Araújo}

Universidade de Fortaleza - UNIFOR, Fortaleza Ceará.

\section{Thelma Leite de Araújo}

Universidade Federal do Ceará, Fortaleza Ceará.

\section{Marta Maria Coelho Damasceno}

Universidade Federal do Ceará, Fortaleza Ceará. 